\title{
SACRAL STRESS FRACTURE IN A RUNNER: A CASE REPORT
}

\author{
doi: 10.1590/S1807-59322009001100014
}

Luciano Miller Reis Rodrigues, Fabrício Hidetoshi Ueno, Edgar Santiago Valesin Filho, Edison Noboru Fujiki, Carlo Milani

\section{INTRODUCTION}

Stress fractures result from cyclic overload and usually affect the bones of the lower extremities. These stress fractures occur most often in athletes and in members of the military. ${ }^{1,2}$ These fractures, which are also known as fatigue fractures, are susceptible to repeated trauma and typically involve the metatarsal bones, femur, tibia, fibula, pelvis or spine. The few cases of sacral stress fractures that have been described in the literature involve young, physically active patients, usually females. ${ }^{3}$

Sacral stress fractures are considered a rare occurrence, and the diagnosis of these fractures is often delayed because the main identifying symptom, lower back pain, is highly prevalent in the general population. ${ }^{4} \mathrm{~A}$ stress fracture should be considered as a possible source of pain if the pain is associated with overuse while walking or running.

Herein, we report the case of a female runner with a stress fracture who complained of lower back pain that was aggravated by performing the single-foot stance.

\section{CASE DESCRIPTION}

We report the case of a 34-year-old woman who complained of lower back pain after exercise. The patient was running five times a week in preparation for a marathon. The training sessions were performed on flat, paved tracks, and the patient was wearing appropriate shoes during the training sessions. The patient did not have a history of hormonal or menstrual disturbances, nor had there been recent increases in the frequency or intensity of the patient's training sessions.

Musculoskeletal System Diseases Department, Faculdade de Medicina do ABC - Santo André / SP, Brazil.

Email: luciano.miller@uol.com.br

Tel: 55114993.5400
The lower back pain was reported to progressively worsen, and the patient was unable to exercise within three weeks from the onset of pain. The patient reported that the pain worsened when the lower right limb made contact with the ground.

The physical examination revealed lower right back tenderness but no changes in the sacroiliac joint and no motor or sensory impairment. The subsequent $\mathrm{x}$-ray analyses were normal. The bone scan showed an increased uptake in the right sacral region (Fig. 1), and magnetic resonance imaging confirmed this finding, showing an area with a hypersignal suggestive of a sacral stress fracture (Fig. 2).

We recommended that the patient refrain from weight bearing exercises and physical activity for 12 weeks, with a gradual return after appropriate rehabilitation. Nine months

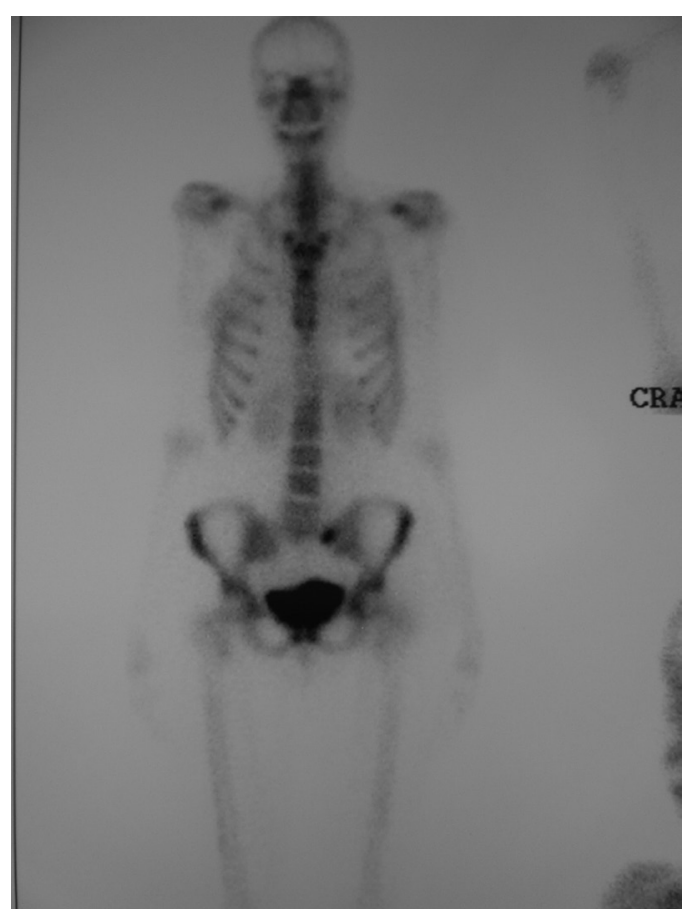

Figure 1 - A bone scan showing the increased uptake in the right sacral region 


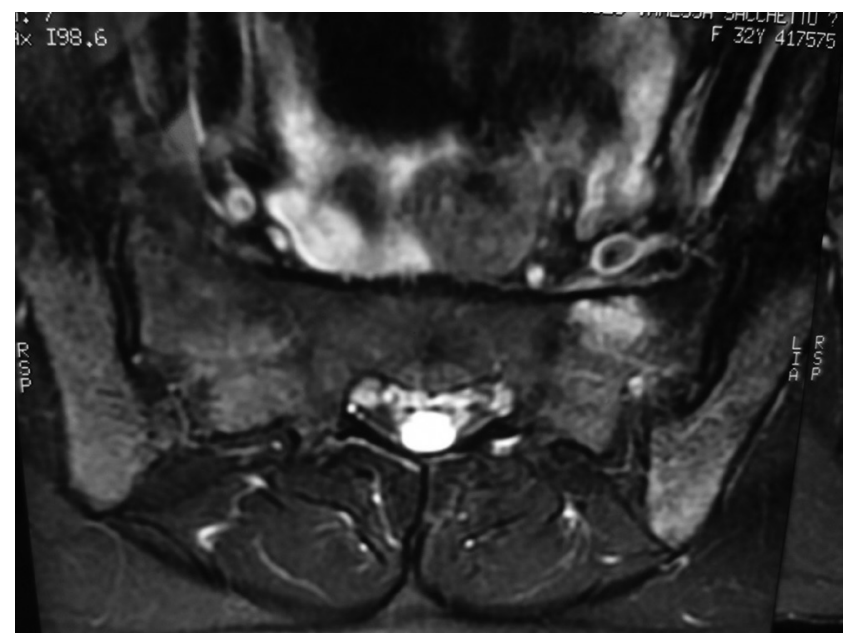

Figure 2 - A magnetic resonance image showing an area with a hypersignal suggestive of a sacral stress fracture

after the fracture, the patient resumed her normal running practice.

\section{DISCUSSION}

Sacral stress fractures may be categorized as either insufficiency or fatigue fractures, depending on the affected population. ${ }^{5-7}$ Insufficiency fractures in general occur in the elderly, in female patients undergoing radiation therapy for the treatment of pelvic neoplastic diseases, or in patients treated with high-dose corticotherapy. ${ }^{8}$ The disorder was initially described by Lourie in $1982^{9}$ as a spontaneous fracture of an osteoporotic bone in post-menopausal women. This type of fracture results from the application of a normal physiological load on a weakened bone with reduced structural or mineral density. In fatigue fractures, there is a skeletal failure resulting from a submaximal repetitive load applied over a prolonged period of strenuous activity. ${ }^{6}$ The incidence rate of sacral fatigue fractures has not been reported in the literature and remains unclear. However, most reports suggest more frequent occurrence in the second and third decades of life.

In women, fatigue fractures are believed to be correlated with nutritional disorders, hormonal disturbances, and amenorrhea. ${ }^{3}$

The main symptom of fatigue fractures in particular is pain in both the lower back and buttocks. A physical examination in patients with fatigue fractures reveals a decreased range of motion in the lower back and tenderness upon palpation of the sacral region. Sacroiliac tests can be performed to assess whether the sacroiliac joint is fractured, although they cannot determine the cause of fracture. No abnormalities were observed in our patient upon completion of these tests, probably because her sacral injury occurred closer to the mid-line, this localization of fracture typically results in a slower and antalgic gait.

Fatigue fractures in the pelvis most often involve the pubic rami, the symphysis and the sacral bone, whereas the sacral ala is more frequently affected in athletes, ${ }^{10}$ as observed in our previous report.

In both insufficiency and fatigue fractures, an important etiological factor is the stress due to the dissipation of vertical force from the trunk onto the sacral bone and sacral alae. ${ }^{5,10}$ Other possible predisposing factors include dissymmetric lower limbs, muscle insufficiency, increased physical demand, genetic and environmental influences, training methods, the use of inappropriate shoes, and changes in pelvic anteversion. ${ }^{6}$

According to Wolff's Law, repeated microtraumas can induce bone remodeling. However, cyclic stress loads induce increased rates of osteoclastic activity, which are associated with increased osteoblastic activity in newly formed bones. Without sufficient rest and time to accumulate newly formed bone, progressive weakness of these bone structures occur resulting in fractures. Interestingly, the process of bone reorganization and repair results in the temporary weakness of the bone prior to the production of a cyclic-overloadresistant final structure. As a general rule, this weakness is observed several weeks after the onset of a training program or during a change in exercise intensity. ${ }^{10}$

According to several authors, magnetic resonance imaging is the method of choice for the diagnosis of fatigue bone fractures. ${ }^{6}$ The typical image includes a low-signal area in T1 with corresponding high-signal areas in T2. Fat suppression sequences may enhance the sensitivity of detecting bone marrow edema, which is suggestive of an acute fracture. Laboratory tests are important in identifying conditions associated with an altered bone metabolism, such as rickets, thyroid dysfunction, or hyperparathyroidism. In addition, biochemical markers of bone formation and resorption such as osteocalcin, bone alkaline phosphatase, and urinary pyridinoline may be used to screen for conditions with altered bone turnover, such as osteoporosis and hormone-related calcium metabolism disorders.

Sacral fatigue fractures typically respond well to rest and changes in the individual's training regimen. ${ }^{3,6,10}$ The goal of treatment is to reduce the number of sessions involving vigorous exercise, especially those exercises involving cyclic weight bearing episodes, a strategy that was employed in the case of this patient. Incidentally, recent studies show an inverse relationship between the use of contraceptives and the occurrence of stress fractures. ${ }^{10}$

Preventive measures include an appropriate choice of shoes, an appropriate running track, medical and nutritional follow-up, hormonal laboratory work-up in female athletes, and avoiding overload during exercise. 


\section{REFERENCES}

1. Shah MK, Stewart GW. Sacral stress fracture: an usual cause of low back pain in an athlete. Spine. 2002;27:E104-8.

2. Major NM, Helms CA. Sacral stress fractures in long-distance runners. AJR. 2000;174:727-9.

3. Lin J, Lachmann E, Nagler W. Sacral insuficiency fractures: a report of two cases and a review of the literature. J Womens Health Gend Based Med. 2001;10:699-705.

4. Bottomley MB. Sacral stress fracture in a runner. Br J Sports Med. 1990;24:243-4.

5. Ahovuo JA, Kiuru MJ, Visuri T. Fatigue stress fracture of the sacrum: diagnosis with MR imaging. Eur Radiol. 2004;14:500-5.
6. Lin JT, Lane JM. Sacral stress fracture. J Womens Health. 2003;12:879 88.

7. Schindler OS, Watura R, Cobby M. Sacral insufficiency fractures. J Orthop Surg. 2007;15:339-46.

8. Tsiridis E, Upadhyay N, Gyannoudis PV. Sacral insufficiency fractures: current concepts of management. Osteoporos Int. 2006;17:1716-25.

9. Lourie H. Spontaneous osteoporotic fracture of the sacrum. An unrecognized syndrome of the elderly. JAMA. 1982;248:715-7.

10. Miller C, Major N, Toth A. Pelvic stress injuries in athlete: management and prevention. Sports Med. 2003;33:1003-12. 